\title{
ÉVALUATION DE L'EFFET DE L'ENVIRONNEMENT ET DE L'ALIMENTATION SUR L'APPARITION D'ANOMALIES DE DÉVELOPPEMENT CHEZ L'ALEVIN DE BAR (DICENTRARCHUS LABRAX).
}

\author{
B. MENU (1), J.H. ROBIN (2), M.F. GOUILLOU-COUSTANS (2)
}

(1) Station IFREMER, Chemin de Maguelone, 34250 Palavas-les-Flots, France.

(2) Unité Mixte de Nutrition des Poissons INRA-IFREMER, Centre IFREMER de Brest, Zl de la pointe du Diable, 29280 Plouzané, France.

\section{RÉSUMÉ}

Une ponte naturelle de bar, obtenue dans les installations expérimentales de Palavas, est mise en élevage dans ce même laboratoire et dans celui de Brest, après un transport fictif ou réel. Les protocoles spécifiques de chacun des deux laboratoires sont utilisés sans changer le contexte d'élevage particulier de chacun des deux sites : mise à jeun à l'obscurité jusqu'à 160 degrés jours et première alimentation sur Artemia à Palavas en bacs de 500 litres et, première alimentation à 75 degrés jours sur rotifères à Brest en bacs de 250 litres et de 35 litres. Dans les conditions d'environnement comparables (bacs de 250 litres à Brest) une période de jeûne est également testée, de 75 à 160 degrés jours, soit en obscurité sans aération, soit sous éclairage continu avec aération. Les malformations des vertèbres, des maxillaires, des opercules (courts et soulevés), la noninflation de la vessie natatoire et la présence de calculs urinaires sont relevées à 3 mois sur tous les lots.

Plusieurs malformations apparaissent inféodées à un site ou un traitement particulier : cristaux urinaires à Palavas, vessies natatoires non fonctionnelles à Brest, maxillaires anormaux en bac de 35 litres. Les opercules soulevés sont observés dans deux installations utilisant des méthodologies différentes mais sont négligeables dans la ligne d'élevage où les tests méthodologiques ont été effectués. Les taux de survie sont significativement influencés par les traitements. La répartition des fréquences des lordoses entre les sites ou les différents traitements peut s'interpréter comme une conséquence de mortalités sélectives. L'association entre les lordoses et les vessies natatoires non fonctionnelles, déjà connue chez le bar, est observée. L'étude des fréquences croisées des malformations montre également que les opercules courts sont 10 fois plus fréquents chez les individus dont les vessies natatoires ne sont pas fonctionnelles. L'étude séparée des populations de juvéniles possédant ou non une vessie natatoire fonctionnelle montre que la répartition des fréquences des opercules courts est indépendante des traitements ou du site d'élevage. Les fréquences élevées des opercules courts sur la majorité des traitements du site de Brest ne peuvent être dissociées des taux importants de non-fonctionnalité des vessies natatoires.

Mots-clés : pisciculture, élevage larvaire, malformations, effets de l'environnement, alimentation. 


\title{
EVALUATION OF ENVIRONMENTAL AND NUTRITIONAL EFFECTS ON \\ DEVELOPMENTAL ABNORMALITIES OF SEA BASS \\ (DICENTRARCHUS LABRAX) JUVENILES.
}

\begin{abstract}
Larvae from a natural spawn of sea bass were distributed into 3 larval rearing lines in 2 distant sites, after simulated or true transportation. The specific rearing methods in the specific rearing environment of each laboratory were used : starvation under darkness for 160 degrees days and first feeding on Artemia (PP) in the Mediterranean laboratory of Palavas ; first feeding on rotifers at 75 degrees days in the laboratory of Brest (Brittany) in 250 liters $(\mathrm{BB})$ tanks or 35 liters tanks $(\mathrm{Bb})$ of a separate rearing line. In the 250 liters tanks in Brest, 2 additional treatments before first feeding on Artemia were tested : starvation up to 160 degrees days under darkness with no bubbling (BP) ; starvation up to 160 degrees days under continuous illumination with bubbling $(\mathrm{BI})$. Vertebral, maxillary, opercular (short or swollen) abnormalities as well as uninflation of the swimbladder and urinary calculi were recorded on 3 months old juveniles in all batches.
\end{abstract}

Some abnormalities appeared to be closely related to a specific site or treatment such as urinary calculi in Palavas, uninflated swimbladder in Brest (maxillary abnormalities in 35 liters tanks). Swollen operculum deformities occurred in two rearing lines subjected to different rearing methods ( $P P$ and $\mathrm{Bb}$ ) but were absent in the rearing line where environmental and nutritional factors were compared. Significant differences in survival rates were observed between treatments. The frequency pattern of lordosis seemed to be the result of selective mortality. Lordosis was often associated with uninflation of swimbladder. Short operculum occurred ten times more often when fish had an abnormal swimbladder. A separate investigation of juvenile populations with or without a normal swimbladder shows that the frequency distribution of the short operculum is independent of treatment and rearing site. The high frequency of short operculum recorded in some treatments cannot be dissociated from the high occurrence of uninflated swimbladder.

Key-words : fish culture, larval rearing, abnormalities, environmental effects, diet.

\section{INTRODUCTION}

Les anomalies de développement sont fréquemment observées chez les juvéniles de poissons marins ou dulçaquicoles élevés en conditions intensives. Ces différences entre phénotypes d'élevage et sauvage constituent un obstacle aussi bien pour le grossissement en captivité (CHATAIN et DEWAVRIN, 1989) que pour le repeuplement (HOWELL, 1994). Dans le cas particulier du bar (Dicentrarchus labrax) les malformations les plus visibles comme les lordoses ou les défauts operculaires ont nettement régressé au fur et à mesure de l'amélioration des méthodes zootechniques (BARAHONAFERNANDES, 1982 ; CHATAIN, 1994a). Si la non-inflation de la vessie natatoire due à des conditions environnementales inadaptées (WEPPE et JOASSARD, 1986) a été clairement identifiée comme la cause la plus fréquente des lordoses observées chez cette espèce (CHATAIN, 1987), les causes de la diminution spectaculaire des malformations operculaires, de $90 \%$ à moins de $5 \%$, restent inconnues.

Les juvéniles de bar issus d'écloseries présentent, encore de nos jours, des anomalies de développement qui portent essentiellement sur le squelette appendiculaire (MARINO et al., 1993), axial (BOGLIONE et al., 1995) ou céphalique (MENU, 1994). De très nombreux facteurs, d'origine environnementale ou génétique, ont été considérés 
comme des causes possibles de ces anomalies chez les téléostéens aussi bien dans le milieu naturel qu'en captivité (DIVANACH et al., 1996). L'étiologie exacte de ces syndromes est paradoxalement mal connue. En élevage intensif, les malformations continuent d'avoir un impact biologique et économique négatif, le seul palliatif consiste à éliminer le plus précocement possible les lots ou les individus anormaux.

Des variations qualitatives et quantitatives des malformations des juvéniles de bar élevés dans les laboratoires IFREMER de Brest et de Palavas ont été observées à plusieurs reprises au cours de ces dernières années. Pour faire une première évaluation de l'influence de conditions d'élevage différentes et de la première alimentation sur les anomalies morpho-anatomiques, un lot de larves de même origine génétique est élevé dans ces deux laboratoires jusqu'à une taille suffisante pour que les anomalies de développement puissent être relevées. Dans les deux laboratoires les méthodologies habituelles d'élevage ont été utilisées ; dans l'un, une comparaison de certaines différences de méthode (alimentation initiale et conditions d'environnement liées à l'alimentation) a été effectuée.

\section{MATÉRIEL ET MÉTHODES}

Une ponte naturelle du stock de géniteurs du laboratoire de Palavas, en décalage avancé de ponte, est répartie en 10 Cubitainers de transport contenant chacun 18 litres d'eau de mer et 100000 oeufs embryonnés. 6 Cubitainers sont expédiés par avion au Centre IFREMER de Brest alors que les 4 derniers, conditionnés de façon identique, subissent un transport fictif de durée équivalente (24 heures) à Palavas.

Les conditions d'élevage en fonction des différents traitements sont synthétisées dans le Tableau I. Les pré-larves séjournent à l'obscurité avant nourrissage à une température la plus voisine possible de $16^{\circ} \mathrm{C}$. A partir de 160 degrés jours, la température des bacs est graduellement montée à $20^{\circ} \mathrm{C}$ et les larves sont nourries de façon identique. Le traitement BB est la méthode classiquement utilisée au laboratoire de Brest : les larves sont alimentées par des rotifères à partir de l'ouverture de la bouche, ce qui nécessite un éclairement et l'homogénéisation du bassin par un bullage. Le traitement BP est une adaptation, en fonction de l'installation utilisée, des conditions de la méthode utilisée à Palavas (PP, COVES et al., 1991). Le traitement intermédiaire BI combine le schéma d'alimentation BP dans les conditions environnementales de BB. Des éléments complémentaires sont obtenus à partir de bacs de 35 litres $(\mathrm{Bb})$ d'une autre ligne d'élevage suivant un schéma d'élevage analogue au traitement BB.

A 610 degrés jours, des larves survivantes sont dénombrées et transférées dans des bacs de sevrage spécifiques des installations à même charge par unité de surface. Les animaux sont sevrés progressivement sur microparticules Kyowa A400 et B400 (Kyowa Hakko Kogyo Co., Japon) distribuées automatiquement pendant la photophase de 12 heures. De 920 degrés jours et jusqu'au bilan final, le granulé Kyowa est progressivement remplacé par un granulé extrudé (Sevbar, INVE, Belgique). Aucun tri n'est effectué sur les lots expérimentaux. Le bilan final a lieu à 3 mois (1510 degrés jours). En fin de manipulation, un échantillon d'alevins est anesthésié au phénoxyéthanol (600 ppm) puis fixé et conservé dans l'eau de mer additionnée de $5 \%$ de formol neutre tamponné. Un relevé individuel du poids, des malformations internes et externes, est effectué sur 50 alevins pour chacune des 4 répétitions des traitements BB et PP, sur 100 individus des 2 répétitions des traitements $\mathrm{BI}$ et $\mathrm{BP}$ et sur 100 individus également pour l'unique bac du traitement $\mathrm{Bb}$. L'ensemble des anomalies est relevé par le même observateur. Les défauts d'inflation de la vessie natatoire, la présence de calculs dans la vessie urinaire et les malformations vertébrales (fusions ou torsions) sont relevés sur des 


\section{Tableau I}

Protocoles utilisés en incubation, en élevage larvaire et au début du sevrage pour les 5 traitements testés sur une même ponte de bar. L'âge est exprimé en degrés jours (DJ).

Table I

Incubation, larval rearing and weaning conditions used for the 5 different treatments applied on the same batch of sea bass. Ages are given in degrees days (DJ)



radiographies, alors que les malformations des opercules courts ou soulevés selon la nomenclature de BARAHONA-FERNANDES (1982) et les malformations des maxillaires sont relevées par observation à l'oeil nu.

Les calculs statistiques utilisés, analyses de variance à un facteur, matrices de corrélation, test $\chi^{2}$ sur tableau de contingence et analyse factorielle des correspondances, sont réalisés avec les logiciels Statgraphics et Unistat (Statistical Graphics Corporation, USA).

\section{RÉSULTATS}

\section{Déroulement}

Au laboratoire de Brest, une crise d'hyperinflation de la vessie natatoire vers le vingtième jour d'élevage entraîne des mortalités importantes. Cette crise d'hyperinflation a touché sélectivement les lots BP et $\mathrm{BI}$, élevés en bacs de 250 litres ayant reçu une première alimentation à 160 degrés jours sur Artemia, épargnant les 4 bacs nourris de rotifères dès l'ouverture de la bouche $(\mathrm{BB})$. Des pics de mortalité ont aussi été observés après 75 degrés jours surtout dans les lots BB et Bl et vers 160 degrés jours surtout dans les lots $\mathrm{BP}$ et $\mathrm{BI}$, mais les récoltes des morts, trop partielles sur des petites larves, ne peuvent fournir de données exploitables. 


\section{Croissance et survie}

\section{Tableau II}

Poids humides formolés, survies en fin d'élevage des larves (1 mois) et à 3 mois, et pourcentage de poissons normaux. A 3 mois figurent les poids moyens des lots non triés et des animaux ne portant aucune malformation. Moyenne et erreur standard par traitement. Les moyennes suivies de lettres en exposant différentes sur la même ligne sont significativement différentes $(P<0,05)$ selon le test de Newman-Keuls, la survie du lot Bb étant exclue $(n=1)$.

\section{Table II}

Wet weights in $\mathrm{mg}$ (formalin preserved) and survival (\%) at the end of larval rearing ( 1 month), weaning ( 3 months) and corresponding proportion of normal fish (\%). At 3 months, weight values are given for whole samples and, in the last line, for the normal fish subclass. Mean and standard error per treatment. Means on the same row with different superscript letters are significantly different ( $P<0.05$, Newman-Keuls test), excluding Bb for survival $(n=1)$.

\begin{tabular}{lccccc}
\hline & PP & BB & BI & BP & Bb \\
\hline Poids à 160 degrés jours (mg) & $0,25 \pm 0,04^{\circ}$ & $0,34 \pm 0,09^{\circ}$ & $0,22 \pm 0,03^{\circ}$ & $0,18 \pm 0,02^{\circ}$ & \\
Survle 0-1 mols (\%) & $22 \pm 5^{\circ}$ & $43 \pm 4^{\circ}$ & $16 \pm 3^{\circ}$ & $34 \pm 2^{\circ}$ & 43 \\
Poids à 1 mols (mg) & $14,3 \pm 0,3^{\circ}$ & $26,3 \pm 0,6^{\circ}$ & $19,6 \pm 0,6^{\circ}$ & $17 \pm 0,4^{\circ}$ & \\
Survie 1-3 mols (\%) & $72 \pm 3^{\circ}$ & $90 \pm 2^{\circ}$ & $88 \pm 2^{\circ}$ & $90 \pm 1^{\circ}$ & 69 \\
Survie 0-3 mols (\%) & $15 \pm 3^{\circ}$ & $39 \pm 4^{\circ}$ & $14 \pm 5^{\circ}$ & $31 \pm 0^{\circ}$ & 30 \\
Poids brut à 3 mois (mg) & $750 \pm 17^{\circ}$ & $841 \pm 16^{\circ}$ & $920 \pm 17^{\circ}$ & $876 \pm 16^{\circ}$ & $768 \pm 27^{\circ}$ \\
Normaux (\%) & $56 \pm 8$ & $35 \pm 15$ & $69 \pm 4$ & $43 \pm 3$ & 9 \\
Poids à 3 mois des juvéniles normaux (mg) & $788 \pm 22^{\circ}$ & $922 \pm 30^{\circ}$ & $954 \pm 18^{\circ}$ & $944 \pm 26^{\circ}$ & $1115 \pm 17^{\circ}$ \\
\hline
\end{tabular}

Les poids moyens obtenus pendant l'élevage des larves sont différents entre traitements (Tableau II). Le poids des larves à l'éclosion est de 0,28 mg. L'alimentation initiale par des rotifères permet d'obtenir une croissance avant 160 degrés jours, le maintien à jeun se traduisant par une perte de poids significativement plus importante pour le traitement BP que pour le traitement BI. Les différences s'estompent au cours du sevrage. Pour les lots élevés dans des environnements comparables pendant les trois mois de l'expérience à Brest $(\mathrm{BB}, \mathrm{BI}$ et $\mathrm{BP})$, on constate que le poids moyen final des juvéniles non malformés n'est pas statistiquement différent. Les différences significatives de survies, à la fin du premier mois d'élevage, conditionnent dans une large mesure les résultats obtenus sur la totalité de la période. On note également une différence significative de plus faible amplitude de 1 à 3 mois entre les lots élevés à Palavas et à Brest.

\section{Etude globale des profils des malformations par traitement et par site}

Le Tableau III montre une très importante hétérogénéité qualitative et quantitative des résultats obtenus sur les sites de Brest et de Palavas. Les lots de Palavas se caractérisent par une prédominance des opercules soulevés, et une importance relative des calculs urinaires. Des différences existent également au laboratoire de Brest entre les lots issus des bacs d'élevage de 250 litres et celui issu des bacs de 35 litres, ces derniers 
induisant des opercules soulevés et des déformations des maxillaires en plus des malformations vertébrales, des absences de vessie natatoire et des opercules courts que l'on retrouve avec des fréquences variables dans les différents traitements en 250 I. Les déformations vertébrales représentent les malformations les plus fréquentes, tous traitements confondus, soit $69 \%$ des animaux anormaux.

\section{Tableau III}

Profils des malformations, anomalies de développement et survies en pourcentages, observés pour chaque réplicat. OPCR : opercule(s) court(s), OPSL : opercule(s) soulevé(s), MAXL : maxillaire(s) malformé(s), CALC : calculs urinaires, VESN : vessie natatoire non fonctionnelle, VTBR : anomalies vertébrales.

\section{Table III}

Abnormalities (\%) and survivals (\%) recorded for every replica. OPCR : short operculum, OPSL : swollen operculum, MAXL : maxillary abnormalities, CALC : urinary calculi, VESN : uninflated swimbladder, VTBR : vertebral abnormalities.

\begin{tabular}{cccccccccc}
\hline Traitements & $\begin{array}{c}\text { Survie à } \\
\mathbf{1} \text { mois }\end{array}$ & $\begin{array}{c}\text { Survile à } \\
\mathbf{3} \text { mols }\end{array}$ & Normaux & OPCR & OPSL & MAXL & CALC & VESN & VTBR \\
\hline PP 1 & 20 & 14 & 48 & 8 & 16 & 4 & 10 & 32 & 26 \\
PP 2 & 20 & 14 & 50 & 2 & 32 & 4 & 10 & 8 & 10 \\
PP 3 & 11 & 9 & 66 & 2 & 16 & 2 & 10 & 12 & 6 \\
PP 4 & 37 & 25 & 60 & 2 & 20 & 0 & 12 & 2 & 14 \\
\hline BB 1 & 40 & 36 & 14 & 20 & 2 & 4 & 2 & 62 & 74 \\
BB 2 & 55 & 51 & 42 & 12 & 2 & 0 & 2 & 18 & 54 \\
BB 3 & 42 & 36 & 36 & 10 & 2 & 0 & 0 & 22 & 58 \\
BB 4 & 34 & 31 & 48 & 12 & 2 & 0 & 0 & 28 & 46 \\
\hline B1 1 & 21 & 19 & 66 & 4 & 4 & 2 & 0 & 2 & 27 \\
BI 2 & 11 & 9 & 72 & 2 & 2 & 1 & 0 & 1 & 25 \\
\hline BP 1 & 35 & 31 & 45 & 6 & 2 & 1 & 0 & 14 & 49 \\
BP 2 & 34 & 30 & 41 & 14 & 3 & 3 & 0 & 25 & 48 \\
\hline Bb & 43 & 30 & 9 & 32 & 21 & 23 & 0 & 85 & 50 \\
\hline
\end{tabular}

Le tableau de contingence (III), croisant les répétitions de tous les traitements avec les malformations, est utilisé pour effectuer une analyse factorielle des correspondances. La représentation simultanée des lignes et des colonnes est donnée en Figure 1 . Les effectifs du cumul individuel des anomalies de développement par traitement, ainsi que les effectifs des malformations regroupées par site, sont utilisés comme éléments supplémentaires. Ces éléments permettent l'identification des axes sans intervenir dans leur calcul (Figure 2). Le premier axe factoriel qui traduit $65 \%$ de la variance oppose le schéma d'élevage réalisé au laboratoire de Palavas à tous ceux testés à Brest. Le second axe qui traduit $28 \%$ de la variance correspond à un classement traduisant la tendance au cumul individuel des malformations. Les lots élevés en bacs de 250 litres à Brest se projettent à droite du graphique. Les lots $\mathrm{BI} 1$ et $\mathrm{BI} 2$, les moins malformés, sont dans la partie haute de l'axe 2. On remarque que les projections des différentes répétitions des traitements $\mathrm{BB}$ et $\mathrm{BP}$ sont très intriquées, ce qui indique une relative homogénéité des réponses qualitatives et quantitatives, en termes de malformations, dans des conditions contrôlées différentes. Le traitement $\mathrm{Bb}$ s'oppose sur le deuxième axe factoriel aux autres traitements réalisés dans le même laboratoire, cette position singulière est due aux fréquences très élevées des malformations et au cumul de malformations. 




Figure 1

Analyse factorielle des correspondances réalisée sur les effectifs des malformations du Tableau Ifl. Représentation simultanée des lignes (répétitions des traitements) et des colonnes (anomalies de développement).

Figure 1

Factorial correspondence analysis based on Table III data. Simultaneous representation of lines (replicates per treatment) and rows (abnormalities). First axis accounts for $65 \%$ of total variance, second axis for $28 \%$.

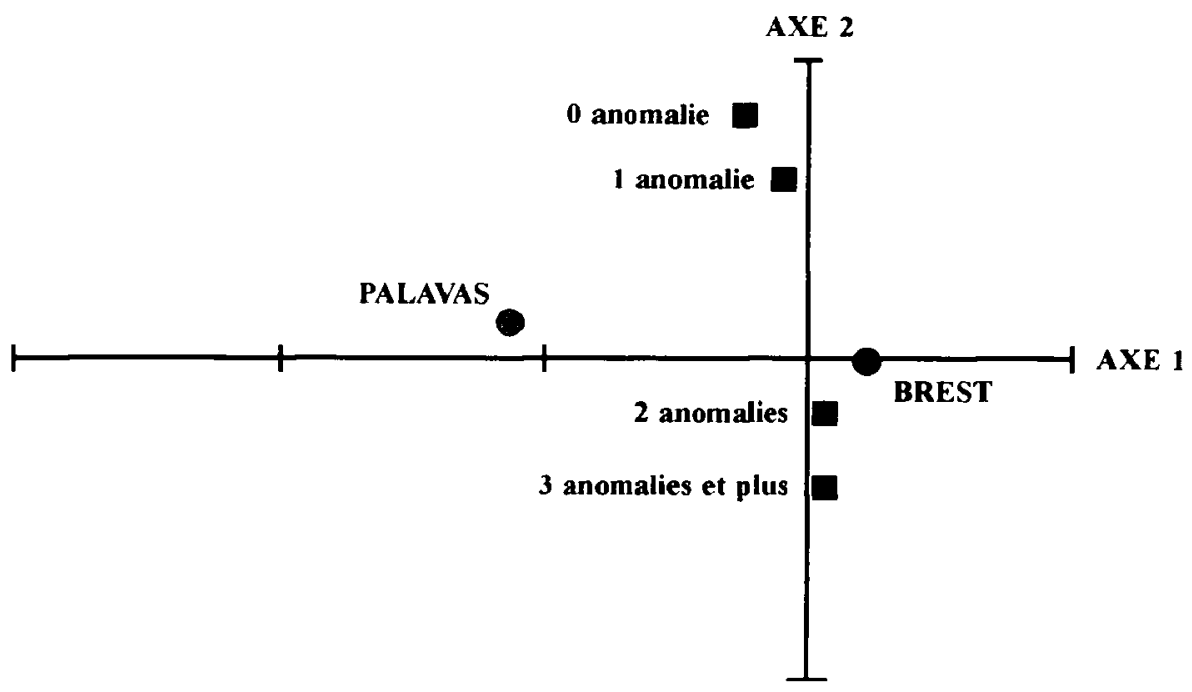

Figure 2

Représentation des éléments supplémentaires, lignes (Brest et Palavas) et colonnes (cumul de malformations) ajoutés aux données initiales de l'analyse factorielle des correspondances de la Figure 1.

Figure 2

Representation of supplementary data added to the factorial correspondence analysis (Figure 1) : rearing site as supplementary rows and number of abnormalities per animal as supplementary columns. 
Les corrélations ont été calculées entre les fréquences de malformation et les survies. Seules les fréquences des malformations vertébrales sont systématiquement corrélées significativement aux survies à 1 mois $(0,75 p=0,003$ en comparant tous les lots, $n=13$ et $0,81 p<0,01$ pour les lots de 250 litres à Brest, $n=8$ ) ainsi qu'aux survies à 3 mois $(0,79 p=0,02$ tous lots et $0,75 p=0,03$ lots de Brest). Des corrélations, entre survies et lordoses, subsistent si l'on considère séparément les animaux ayant une vessie natatoire fonctionnelle et ceux n'en ayant pas $(+0,83$ et $+0,76$ respectivement $)$. Les opercules courts paraissent corrélés aux survies, ce n'est significatif que pour l'ensemble des lots à 1 mois $(+0,61 p<0,05)$. Les corrélations entre les survies et les vessies natatoires non fonctionnelles ne sont pas significatives. Les malformations des maxillaires, les opercules soulevés et les calculs urinaires ne sont pas corrélés aux survies.

\section{Etude des lots issus des bacs d'élevage larvaire de 250 litres à Brest}

L'effet du schéma alimentaire a été abordé pour les élevages larvaires menés en bacs de 250 litres à Brest où ont été testés l'alimentation initiale et les facteurs d'environnement nécessaires à l'alimentation et qui subissent ensuite des conditions identiques. Les taux de vessie natatoire non fonctionnelle, d'opercule(s) court(s) et d'anomalies vertébrales sont élevés chez les juvéniles issus de 2 des 3 traitements (Tableau III). L'étude du cumul individuel des malformations sur les 600 individus montre que ces 3 malformations ne sont pas indépendantes. Les anomalies vertébrales sont 2 fois plus fréquentes et les opercules courts 10 fois plus fréquents chez les individus dont la vessie natatoire n'est pas fonctionnelle (Tableau IV). Les calculs de $\chi^{2}$ effectués sur ces données montrent alors que les traitements n'ont plus d'effet significatif sur les fréquences d'opercules courts observés. Une généralisation de cette constatation est possible pour la totalité des traitements effectués sur les 2 sites, avec la prudence qu'imposent les effectifs modestes observés sur le site de Palavas. Un effet significatif des traitements subsiste sur les malformations vertébrales, surtout si l'on considère celles non liées à une vessie natatoire anormale, avec un effet site hautement significatif. Les calculs de $\chi^{2}$ portant sur les fréquences des anomalies d'inflation de la vessie natatoire différencient les lots $\mathrm{B}$ inférieurs aux deux autres traitements, mais une différence isole une répétition du traitement $\mathrm{BB}$ des autres lots $\mathrm{BB}$ et $\mathrm{BP}$.

\section{Tableau IV}

Répartition des anomalies vertébrales et des opercules courts suivant l'état de fonctionnalité de la vessie natatoire, avec indication des probabilités des valeurs de $\chi^{2}$ correspondantes. L'hypothèse nulle est l'homogénéité.

\section{Table IV}

Distribution of vertebral abnormalities and short opercula among juveniles with normal swimbladder or uninflated swimbladder, and significant differences for $\chi^{2}$ test. Null hypothesis is homogeneity.

\begin{tabular}{|c|c|c|c|c|}
\hline & \multicolumn{2}{|c|}{ Vessie natatoire normale } & \multicolumn{2}{|c|}{ Vessie nataloire anormale } \\
\hline & $\begin{array}{l}\text { Anomalies } \\
\text { vertébrales }\end{array}$ & $\begin{array}{l}\text { Opercules } \\
\text { courts }\end{array}$ & $\begin{array}{c}\text { Anomalies } \\
\text { vertébrales }\end{array}$ & $\begin{array}{l}\text { Opercules } \\
\text { courts }\end{array}$ \\
\hline BB & $44 \%$ & $5 \%$ & $86 \%$ & $31 \%$ \\
\hline $\mathrm{BI}$ & $26 \%$ & $3 \%$ & $(n<5)$ & $(n<5)$ \\
\hline $\mathrm{BP}$ & $42 \%$ & $3 \%$ & $74 \%$ & $38 \%$ \\
\hline Moyenne pondérée & $36 \%$ & $3 \%$ & $80 \%$ & $34 \%$ \\
\hline Probabilité $\chi^{2}$ & $* *$ & NS & $\begin{array}{c}\mathrm{NS} \\
\text { (BB et } \mathrm{BP})\end{array}$ & $\begin{array}{c}\text { NS } \\
(B B \text { et BP })\end{array}$ \\
\hline Site de Palavas & $9 \%$ & $1 \%$ & $48 \%$ & $19 \%$ \\
\hline Site de Brest & $36 \%$ & $4 \%$ & $69 \%$ & $35 \%$ \\
\hline Probabilité $\chi^{2}$ & $* \star \star$ & NS & 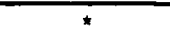 & NS \\
\hline
\end{tabular}




\section{DISCUSSION}

\section{Malformations des vertèbres, vessies natatoires et opercules courts}

En première analyse, l'étude comparée des profils des anomalies de développement par traitement et par site montre une certaine spécificité des types et des fréquences de malformations dans les installations des deux laboratoires. L'existence de certaines corrélations doit être prise en compte pour mieux appréhender les résultats. On constate ainsi que les distributions des fréquences d'anomalies de l'axe vertébral et des vessies natatoires et, de l'axe vertébral et des opercules courts ne sont pas indépendantes. La relation de cause à effet entre la non-inflation des vessies et les lordoses a été montrée chez le bar (CHATAIN, 1994b). Le fait que les opercules courts apparaissent dix fois plus fréquemment chez les alevins ne possédant pas une vessie natatoire fonctionnelle est un apport de la présente expérience.

Les fréquences des lordoses avec ou sans vessie natatoire fonctionnelle sont fortement corrélées aux taux de survie à 3 mois et plus précisément aux survies à 1 mois. Ces résultats suggèrent fortement que des mortalités différentielles centrées sur le premier mois d'élevage soient responsables des différences observées sur ce critère et ceux qui y sont liés. Le syndrome associant les opercules courts, les lordoses et la non-inflation de la vessie natatoire, qui paraissait, en première analyse, si spécifique de Brest, peut s'interpréter, pour les deux sites, comme un problème primordial d'inégalité d'inflation des vessies natatoires, de corrélations entre malformations et d'effet sélectif des mortalités. Les malformations des opercules de $30 \%$ de la population ayant des vessies natatoires anormales dans les alevins élevés à Brest, restent très inférieures aux 70 à $90 \%$ des populations totales observés par BARAHONA-FERNANDES $(1978,1982)$. Les taux de survie larvaire décrits par cet auteur, comparables aux nôtres, ne permettent pas une interprétation faisant intervenir des mortalités différentielles.

Les deux types d'environnements abiotiques (BB et $B \mid$ versus $B P$ ) et les deux schémas d'alimentation (BP et $B \mid$ versus $B B$ ) n'ont donc une influence significative que sur la répartition des fréquences des vessies natatoires non fonctionnelles. Les meilleurs résultats sur ce critère anatomique sont obtenus sur le lot $\mathrm{BI}$, maintenu à jeun sous un éclairement de 300 lux. Ce résultat semble être en contradiction avec ceux de WEPPE et JOASSARD (1986) qui obtenaient leur plus mauvais taux d'inflation avec les lots maintenus à une intensité d'éclairement de 300 lux. Mais cette contradiction apparente peut être l'effet du faible taux de survie pour le lot BI. Dans la même ligne d'élevage, l'alimentation avec des rotifères (BB) permet logiquement une croissance plus précoce que les lots maintenus à jeun (BI et $\mathrm{BP}$ ), mais permet également d'éviter une crise d'hyperinflation. L'hyperinflation de la vessie natatoire est une pathologie liée au stress (JOHNSON et KATAVIC, 1984). KATAVIC (1986) suggère que le syndrome puisse être causé par des stress d'ordre nutritionnel. Dans la présente expérience, le stress induit par une période de jeûne peut être mis en cause dans l'apparition du syndrome. Cet effet peut être aggravé par les conditions d'environnement initiales (lumière continue et aération) du traitement $\mathrm{BI}$, qui présente des survies significativement inférieures.

\section{Malformations spécifiques de site ou de traitements et non corrélées aux survies}

Un effet de l'environnement d'élevage, au sens large, est montré sur les fréquences d'apparition des opercules soulevés et des malformations des maxillaires dans les bacs de 35 litres à Brest, des opercules soulevés et des calculs urinaires sur le site de Palavas. Les opercules soulevés ont été observés avec deux méthodes d'élevage très différentes dans ces deux installations alors qu'ils ne sont apparus qu'à des taux négligeables dans 
l'installation de 250 l. Pour ces malformations non corrélées aux survies, on retrouve la notion de "mélange original de malformation " suggérée par BOGLIONE et al. (1994) après une étude de la qualité des productions de plusieurs fermes marines italiennes. L'uniformité du matériel biologique utilisé dans notre expérience permet, en outre, d'écarter l'hypothèse que ces différences soient liées à la qualité de la ponte. La grande disparité des conditions d'élevage entre les deux sites ne permet pas d'identifier l'origine de ces différences. La spécificité des principales malformations, rencontrées sur chaque site pendant cette expérience, correspond bien aux observations déjà effectuées lors d'autres élevages. Par contre, les fortes fréquences d'anomalies, ici obtenues, sont exceptionnelles : un effet de la qualité de la ponte sur l'ampleur du phénomène est probable Par ailleurs, l'association entre les cristaux urinaires et la non-inflation de la vessie natatoire décrite par BOGLIONE et al. (1995) n'a pas été retrouvée dans nos résultats.

\section{CONCLUSION}

A partir d'une même ponte, différentes anomalies du juvénile de bar ont été observées à des taux très différents selon les installations et les traitements utilisés. Chaque installation semble induire un mélange typé de malformations. Dans une même ligne d'élevage, des facteurs contrôlés (alimentation différée ou non, environnement pendant une phase de jeûne) n'ont pas semblé agir sur le type local des malformations majoritaires (anomalies vertébrales, vessie natatoire non fonctionnelle et opercules courts), mais plutôt sur leurs fréquences en fonction des survies. La survie est principalement corrélée aux malformations vertébrales. Ceci peut masquer d'éventuels effets des traitements sur l'inflation des vessies natatoires, car ces deux malformations sont reliées. Les opercules courts sont surtout rencontrés chez les alevins ayant une non-inflation de la vessie natatoire. Cette relation explique, en partie seulement, la plus faible fréquence d'opercules courts rencontrée depuis que l'amélioration des techniques permet de meilleurs taux d'inflation de la vessie natatoire chez les larves de bar.

\section{REMERCIEMENTS}

Les auteurs remercient les équipes techniques des deux laboratoires et particulièrement Marie-Odile VIDAL et Jean-Claude ALEXANDRE pour leur précieuse collaboration.

\section{BIBLIOGRAPHIE}

BARAHONA-FERNANDES M.H., 1978. L'élevage intensif des larves et des juvéniles du bar (Dicentrarchus labrax (L.)), données biologiques, zootechniques et pathologiques. Thèse de Doctorat d'Etat, Université d'Aix-Marseille II, France, 208 p.

BARAHONA-FERNANDES M.H., 1982. Body deformation in hatchery reared European sea bass Dicentrarchus labrax (L). Types, prevalence and effect on fish survival. Journal of Fish Biology, 2, 239-249.

BOGLIONE C., MARINO G., FERRERI F., FINOA M.G., SCARDI M., FRESI E., CATAUDELLA S., 1994. Anatomical aspects for seed quality assessment in sea bass (Dicentrarchus labrax), hatchery and wild populations. In MUIR J. and SEVILA F. (eds), Measures for success, 191-197, EAS Spec. Pub. $n^{\circ} 21$, Oostende, Belgium. 
BOGLIONE C., MARINO G., FUSARI A., FERRERI A., FINOIA M.G., CATAUDELLA S., 1995. Skeletal anomalies in Dicentrarchus labrax juveniles selected for functional swimbladder. ICES marine Science Symposium, 201, 163-169.

CHATAIN B., 1987. La vessie natatoire chez Dicentrarchus labrax et Sparus auratus. II. Influence des anomalies de développement sur la croissance de la larve. Aquaculture, 65, 175-181.

CHATAIN B., 1994a. Estimation et amélioration des performances zootechniques de l'élevage larvaire de Dicentrarchus labrax et de Sparus auratus. Thèse Doctorale ès Sciences, Université d'Aix-Marseille II, 199 p.

CHATAIN B., 1994b. Abnormal swimbladder development and lordosis in sea bass (Dicentrarchus labrax) and sea bream (Sparus aurata). Aquaculture, 97, 169-180.

CHATAIN B., DEWAVRIN G., 1989. Influence des anomalies de développement de la vessie natatoire sur la mortalité de Dicentrarchus labrax au cours du sevrage. Aquaculture, 78, 55-61.

COVES D., DEWAVRIN G., BREUIL G., DEVAUCHELLE N., 1991. Culture of sea bass (Dicentrarchus labrax L.). In J.P. MAC VEY (ed.), CRC Handbook of mariculture, CRC Press Vol. II, Finfish Aquaculture, 3-20, Boca Raton, Florida, USA.

DIVANACH P., BOGLIONE C., MENU B., KOUMOUNDOUROS G., KENTOURI M., CATAUDELLA S., 1996. Abnormalities in finfish mariculture, an overview of the problem, causes and solutions. In Handbook of contributions and short communications presented at the international Workshop on "Sea bass and Sea bream culture, problems and prospects ", Verona, Italy, October 16-18, 1996, 45-66, EAS, Oostende, Belgium.

HOWELL B.R., 1994. Fitness of hatchery-reared fish for survival in the sea. Aquaculture and Fisheries Management, 25, supplement 1, 3-17.

JOHNSON W., KATAVIC I., 1984. Mortality, growth and swimbladder syndrome of sea bass (Dicentrarchus labrax) larvae under varied environmental conditions. Aquaculture, 38, 67-78.

KATAVIC I., 1986. Diet involvement in mass mortality of sea bass (Dicentrarchus labrax) larvae. Aquaculture, 58, 45-54.

MARINO G., BOGLIONE C., BERTOLINI B., ROSSI A., FERRERI F., CATAUDELLA S., 1993. Observations on development and anomalies in the appendicular skeleton of sea bass, Dicentrarchus labrax L. 1758, larvae and juveniles. Aquaculture and Fisheries Management, 24, 445-456.

MENU B., 1994. Effects of micropellets used as a first larval feed, on the rate of skeletal abnormalities in sea bass and sea bream. In MUIR J. and SEVILA F. (eds), Measures for success, 160-161, EAS, Spec. Pub. n²1, Oostende, Belgium.

WEPPE M., JOASSARD L., 1986. Preliminary study, effects of light on swimbladder's inflation of cultured sea bass. In VIVARĖS C.P., BONAMI J.R. and JASPERS E. (eds), Pathology in marine aquaculture, 379-380, EAS, Spec. Pub. $n^{\circ} 9$, Bredene, Belgium. 